\title{
Tendência secular de crescimento em Blumenau (SC)
}

\author{
Secular trend of growth in Blumenau, Santa Catarina State
}

\author{
Ana Silveira Soncini ${ }^{1}$ \\ Deisi M aria Vargas ${ }^{2}$ \\ M ariana Garcia Lopes Arena ${ }^{3}$ \\ Luiz Fernando Garcia LopesArena ${ }^{4}$
}

${ }^{1}$ Departamento de

M edicina, Fundação

Universidade Regional de

Blumenau. Av. Antonio da

Veiga 140, Vila Nova

89012-900 Blumenau SC.

anasoncini@hotmail.com

${ }^{2}$ Departamento de

Medicina, Centro de

Ciências da Saúde, Fundação

Universidade Regional de

Blumenau.

${ }^{3}$ Faculdade de M edicina de

Itajubá.

${ }^{4}$ Fundação Universidade

Regional deBlumenau.
Abstract Secular trend of growth refers to any change of the corporal size in determined population group in long periods of time. The objective of this work isto study the secular tendency of growth in natural height among recruits in Blumenau, Santa Catarina State, between the years of 1963 and 2007. This is a transversal, retrospective and analytical study. Young recruits, aged 18 to 20 were chosen as the population. A standardized database was used on individual records with the first 40 records of each year being selected. D ata from 1963 to 2007 were collected and separated per decades. A margin of error not higher than 3.5\% was used as a demonstration, which resulted in a sample of 600 individuals. The t-test was used to compare theaverages of different decades. Theresults showed an increase of $7 \mathrm{~cm}$ in the height of the population in Blumenau in the last 50 years. The positive trend that is occurring in our country in the most recent evaluations can be attributed to better sanitary, economic and social conditions. The secular tendency of growth in height was positive in the municipality of Blumenau. It was found that the population increased $7 \mathrm{~cm}$ in the final height in the last 50 years with a growth rate of $0.14 \mathrm{~cm} /$ year or $1.4 \mathrm{~cm} /$ decade.

Key words Growth, Natural height, Military
Resumo Tendência secular do crescimento referese a qualquer mudança do tamanho corporal em determinado grupo populacional em longos períodos de tempo. 0 objetivo deste trabalho é estudar a tendência secular de crescimento em estatura dos recrutas de Blumenau (SC), entre 1963 e 2007. Trata-se de um estudo transversal, retrospectivo e analítico. Como população, foram escoIhidos recrutas com idade entre dezoito e vinte anos. Utilizou-se um banco de dados padronizados em fichas individuais e assim foram selecionadas as primeiras quarenta fichas de cada ano sele cionado. Foram coletados dados de 1963 a 2007 e separados por décadas. Para o cálculo amostral, utilizou-se uma margem de erro não superior a $3,5 \%$, o que resultou em uma amostra de seiscentos inscritos. U tilizou-se 0 teste t-Student para comparar as médias das diferentes décadas. N otase que em Blumenau ocorreu um aumento da ordem de sete centímetros na estatura da população nos últimos cinquenta anos. A tendência positiva que vem ocorrendo em nosso país nas avaliações mais recentes pode ser atribuída a melhorias nas condições sanitárias, econômicas e sociais. A tendência secular de crescimento em estatura foi positiva no município. Verificou-se que a população nos últimos cinquenta anos aumentou sete centímetros na estatura final, tendo como taxa de crescimento $0,14 \mathrm{~cm} /$ ano ou $1,4 \mathrm{~cm} /$ década.

Palavras-chave Crescimento, Estatura, M ilitares 


\section{Introdução}

Tendência secular do crescimento (TSC) referese a qualquer mudança do tamanho corporal ou de sua composição em determinado grupo populacional em longos períodos de tempo. Essas mudanças, quando positivas, não estão associadas basicamente a fatores genéticos, mas sim a fatos sociais, como a normatização do trabalho infantil, tamanho das famílias, alterações do comportamento infantil e condições de saúde individual epopulacional ${ }^{1}$.

A velocidade do crescimento e a altura alcançada nas diferentes idades são fenótipos condicionados pela herança genética dos indivíduos, ou seja, cada indivíduo nasce com um determinado potencial de crescimento que é definido pelo genótipo herdado de seus pais biológicos. A realização plena desse potencial, entretanto, depende da existência de um ótimo estado nutricional e, por extensão, de um bom estado de saúde, de uma alimentação adequada e de boas condições de vida em geral².

Os principais trabalhos sobre o tema revelam que a tendência secular do crescimento tem sido atribuída, sobretudo, a influências ambientais, em particular, a melhorias de condições sanitárias, econômicas e sociais. Esse fenômeno pode ser positivo, negativo ou, ainda, estar ausente 3 .

Os estudos detendência secular de crescimento corporal têm sido importante instrumento para avaliar a trajetória da saúde física de populações, bem como a existência de desigualdades sociais entre diferentes grupos humanos ${ }^{4}$.

É interessante a constatação de que, mesmo nospaíses subdesenvolvidoseem desenvolvimento, em trabalhos realizados com grupos populacionais mais favorecidos, a tendência secular se mostra positiva, em contraste com o que ocorre com o restante da população não favorecida ${ }^{1}$.

N o Brasil, são escassos os estudos enfocando a evolução da estatura média do adulto. Por esta razão, alguns autores têm procurado compensar a escassez de trabalhos sobre tendência secular através da análise de dados baseados em estudos transversais ${ }^{5}$.

0 presente estudo tem como objetivo geral estudar a tendência secular de crescimento em estatura no município de Blumenau (SC). Como objetivo específico, coletar dados em estatura dos recrutas naturais de Blumenau do 23을 Batalhão de Infantaria de Blumenau, entre 1963 e 2007, calcular as médias de estatura por década ecompará-las e, por fim, determinar a taxa de ganho médio em estatura no período estudado.
Material emétodos

Realizou-se um estudo transversal, retrospectivo e analítico. Como população, foram escolhidos os jovens recrutas do 23 을atal hão de Infantaria de Blumenau (SC), incorporados a esta instituição no período de 1963 a 2007, com idade entre dezoito e vinte anos, naturais de Blumenau (SC).

Sendo obrigatório o serviço militar em nosso país, todo jovem do sexo masculino apresentase a uma Junta de Serviço M ilitar no ano em que completa dezoito anos. Devido às implicações legais decorrentes do não comparecimento, 0 al istamento é quase universal para os homens nesta faixa etária. Sendo assim, há pouca seleção no alistamento, restrita a portadores de grave deficiência física e/ou mental, internos em instituições e residentes no exterior, entre outros ${ }^{1}$. M esmo havendo este viés, essa fonte constitui-se em uma importante alternativa para avaliar a TSC ${ }^{6}$.

$\mathrm{Na}$ ocasião do comparecimento do jovem à junta militar, é expedida a Ficha de Alistamento Militar, na qual ficam registrados alguns traços fenotípicos (estatura, cor da pele, dos olhos e do cabelo) e características sociodemográficas (Iocal de nascimento, escolaridade, profissão).

De cada década (por exemplo, 1950-1959), foram analisados três anos medianos: os terminados em 3, 5 e 7. Esta técnica foi escol hida para evitar que fossem coletados dados de anos muito próximos, de décadas diferentes, o que poderia acarretar uma falsa interpretação dos resultados para aquelas décadas (por exemplo: 1959 e 1961). As fichas estavam fora de qualquer tipo de ordem sistemática, separadas apenas por ano de incorporação à instituição, dentro de um arquivo. U tilizou-se uma margem de erro não superior a 3,5\% , cuja população ficou aproximada em três mil indivíduos, que resultou em uma amostra de seiscentos inscritos uniformemente distribuídos nos anos pesquisados. Dessa forma, foram selecionadas as primeiras quarenta fichas do ano estudado, de forma sistemática, excluindo-se apenas as fichas de recrutas procedentes de outro município que não Blumenau (SC). Foram considerados elegíveis somente fichas dealistamento dejovens entre dezoito evinte anos nascidos na cidade de Blumenau.

A coleta foi realizada tendo como referência o banco de dados padronizado em fichas individuais, sem uma ordem sistemática (ordem alfabética, ordem de ingresso na instituição, data de nascimento, ordem crescente ou decrescente de estatura ou de qualquer outra característica individual) contendo as seguintesinformações: dis- 
trito de procedência, data de incorporação à instituição, data e local de nascimento, estatura em centímetros (com precisão decimal) e, em algumas fichas, grau de escolaridade.

As fichas encontravam-se em um arquivo localizado no 23음atalhão de Infantaria de Blumenau (23BI). Sabe-se que, nessa instituição, a partir de 1990, a altura dos recrutas passou a ser medida com um antropômetro de metal (precisão $0,1 \mathrm{~cm}$ ), localizado numa balança mecânica, na qual se faz a medição do peso (precisão 100 g). Essas medidas são feitas com indivíduo descalço ena presença deum médico. Antes de 1990, não se têm dados de como eram coletados peso e altura. Para a análise estatística, utilizou-seo testet-Student e a equação de regressão linear simples. $\mathrm{Na}$ comparação das médias de estatura entre as décadas, testet-Student, optou-se por utilizar os anos terminados em 5 . Com os resultados, foi realizada uma planilha eletrônica de dados e gráficos no programa M icrosoft ${ }^{\circledR}$ Office Excel 2003. Os resultados foram expressos com estatística descritiva. Considerou-sediferença significante os valores de $p \leq 0,05$. Para o cálculo do z-score, utilizou-se valores de referência do $\mathrm{Na}$ tional Center for H ealth Statistics (N CHS) 2000.

0 presentetrabal hado foi protocolado eaprovado pelo Comitê de Ética da Fundação Universidade Regional de Blumenau (FURB).

\section{Resultados}

A tendência das médias de estatura para Blumenau como um todo demonstrou que ocorreu um aumento da ordem de sete centímetros nos últimos cinquenta anos ( $1,4 \mathrm{~cm} /$ década ou $0,14 \mathrm{~cm} /$ ano). A evolução da estatura ao longo desses anos apresentou intensidades distintas de acordo com cada ano selecionado para o estudo. Com o uso da equação da regressão linear simples, verificase uma correlação positiva e forte $r=87,23 \%$, mostrando assim uma estimativa positiva elinear da tendência secular do crescimento da população estudada.

As médias das alturas de cada ano e década também foram calculadas, confirmando assim a tendência secular positiva em estatura, bem como as variações em centímetros, por década (Tabela 1). Dessa forma, observa-se que a tendência secular foi ausente entre as décadas de oitenta e noventa, se analisadas isoladas, porém com um total de sete centímetros no aumento da estatura final da população nosúltimos cinquenta anos.
$\mathrm{Na}$ Tabela 2, são representados os valores da média, do desvio padrão, do coeficiente de variação e intervalo de confiança da altura dos recrutas selecionados no estudo, analisados por décadas.

\section{Discussão}

Os dados existentes em toda a literatura, incluindo os estudos nacionais, deixam inúmeros questionamentos relativos à tendência secular. A tendência positiva quevem ocorrendo em nosso país nas avaliações mais recentes com indivíduos adultos também vem acontecendo na faixa etária pediátrica, principalmente nos escolares, e provavelmente há correlação.

Os aumentos observados em estatura comumente têm sido atribuídos a melhorias nas condições sanitárias, econômicas e sociais. No entanto, influências genéticas já foram postuladas. Para a mai oria das populações, contudo, os dois fatores exógenos mais importantes que influenciam o crescimento são possivelmente a nutrição ea presença/ausência de doenças.

Uma vez em que os países possam estar em períodos de diferente crescimento econômico, grau de instrução da população, campanhas governamentais, entre outros fatores externos que influenciam na estatura final da população, os resultados finais da tendência secular do crescimento, quando comparados, podem trazer uma falsa impressão de que um país têm melhores

Tabela 1. Distribuição das médias das alturas por ano e década, e cálculo do z-score das alturas médias das décadas.

\begin{tabular}{cccc}
\hline Ano & $\begin{array}{c}\text { Altura média } \\
\text { do ano }(\mathrm{m})\end{array}$ & $\begin{array}{c}\text { Altura média } \\
\text { da década }(\mathrm{m})\end{array}$ & z-score \\
\hline 1963 & 1,68 & & $-1,04$ \\
1965 & 1,68 & 1,7 & \\
1967 & 1,73 & & $-0,89$ \\
1973 & 1,7 & & \\
1975 & 1,72 & 1,71 & $-0,28$ \\
1977 & 1,72 & & \\
1983 & 1,75 & & $-0,28$ \\
1985 & 1,75 & 1,75 & \\
1987 & 1,75 & & \\
1993 & 1,76 & & 0,03 \\
1995 & 1,74 & 1,75 & \\
1997 & 1,74 & & \\
2003 & 1,77 & & \\
2005 & 1,78 & 1,77 & \\
2007 & 1,76 & & \\
\hline
\end{tabular}




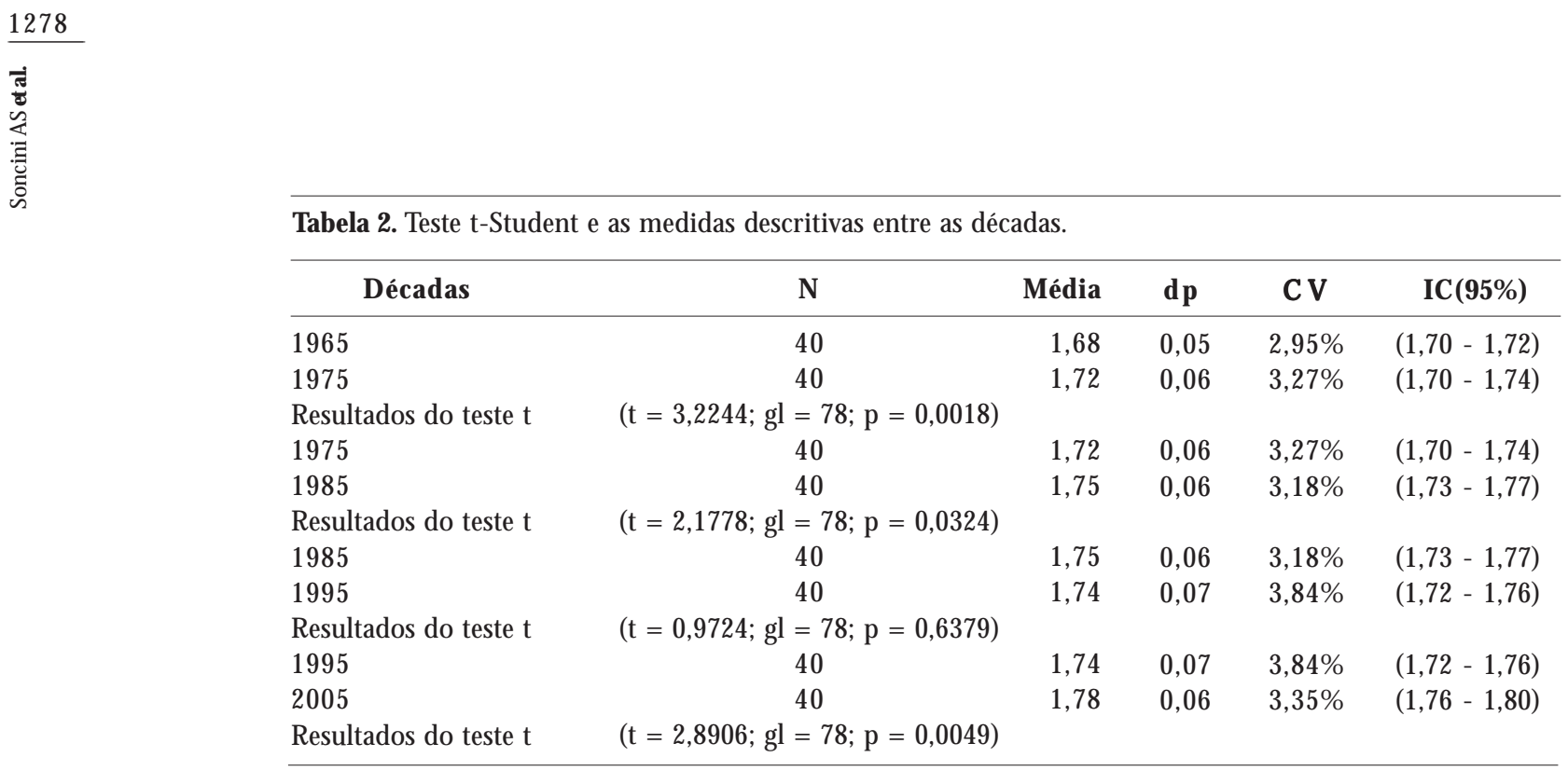

condições que outros, visto que sua TSC possa ser maior. Porém, isso deve ser criteriosamente analisado, uma vez que al guns países já al cançaram quase 0 máximo da altura esperada para sua população, tendo assim uma tendência secular ausente ou levemente positiva.

A ausência de tendência secular pode decorrer de duas situações. Primeiro, o alcance por parte da população do seu potencial genético máximo de crescimento no que tange ao tamanho e tempo de maturação; segundo, condições ambientais insatisfatórias para permitir a ocorrência positiva de tendência secular ${ }^{3}$.

Evidências da eliminação de diferenças na estatura entre classes sociais foram descritas apenas em países cujo desenvolvimento social éhoje de alto nível, como a Noruega, tornando a experiência deste país extremamente peculiar. Já na Grã-Bretanha, por exemplo, apesar do elevado nível médio de vida, diferenças em estatura entre classes sociais ainda persistem ${ }^{6}$.

N os últimos 150 anos, observou-se tendência progressiva para um aumento na estatura de indivíduos nos países industrializados ocidentais e em alguns países em desenvolvimento ${ }^{7}$. As taxas médias, sobretudo para populações européias, variam conforme a idade e o estrato socioeconômico, alcançando em torno de 1,0 a 1,3 cm/ década para crianças en tre cinco edez anos; 1,9 a $2,5 \mathrm{~cm} /$ década durante a adolescência; e 0,6 a 1,0 $\mathrm{cm} /$ década no início da idade adulta ${ }^{3}$. Se este estudo for comparado à taxa média alcançada em Blumenau da década de sessenta até o final da década de oitenta, verifica-se que essa taxa foi de $1,66 \mathrm{~cm} /$ década no início da idade adulta.

Trabalhos realizados em países desenvolvidos, como Estados Unidos, Canadá, Japão e Noruega, sugerem que, entre os anos de 1860 e 1960, ocorreu aumento estatural de $1,3 \mathrm{~cm}$ por década na infância (meninas de oito anos e meninos de dez anos), $1,9 \mathrm{~cm} /$ década na fase intermediária da adolescência (meninas aos doze e meninos aos catorze anos) e $0,6 \mathrm{~cm} /$ década na idade adulta ${ }^{8}$. Este estudo foi importante, porém não se tem estudos em Blumenau sobre TSC no mesmo período para se fazer algumas comparações em relação à tendência secular do crescimento.

Importantes análises de dados em estatura média de recrutas de países europeus no período de 1960 a 1975 podem ser encontradas. 0 que se evidenciou para todos os países foi um considerável aumento na estatura média dos recrutas ao longo do tempo. Esse aumento ocorreu, no entanto, de forma bastante diversa entre os vários países. Os recrutas holandeses foram os queapresentaram a maior estatura média no final do período $(180,1 \mathrm{~cm})$, seguidos dos recrutas sue$\cos (178,4 \mathrm{~cm})$. Para estes países, a tendência foi, respectivamente, de 1,37 e 0,99 $\mathrm{cm} /$ década. Em um bloco intermediário, com ten dências de 0,88 $\mathrm{cm} /$ década, estão a N oruega e a França. As tendências para os demais países estudados foram as seguintes: Bélgica (0,74 cm/década), I tália (0,64 $\mathrm{cm} /$ década) e Dinamarca $(0,53 \mathrm{~cm} / \text { década) })^{9}$. Quando se observa a estatura média dos recrutas de Blumenau na década de sessenta e setenta, tem-ser espectivamentecomo estatura média 1,70 $\mathrm{cm}$ e $1,71 \mathrm{~cm}$ everifica-se uma taxa decrescimento de $1 \mathrm{~cm} /$ década neste período. Esta positividadenaTSC nesteperíodo podeser associada, principalmente, ao "milagre brasileiro", período de mudanças socioeconômicas e culturais do país.

Estudos demonstraram tendência secular de crescimento desde a metade do século XX, com 
aceleração nasúltimas décadas, porém, ainda sem alcançar a expressão completa da sua genética estatural ${ }^{7}$. 0 mesmo pode ser dito desse trabaIho realizado em Blumenau. De acordo com esses estudos, 0 aumento na altura média dos brasileiros nascidos em 1967 em relação aos nascidos em 1952 foi de $0,8 \mathrm{~cm}$ por década. Em todas as regiões brasileiras e em ambos os sexos, a tendência secular de crescimento estatural foi francamente positiva, embora ainda ocorra uma distância significativa entre as alturas dos brasileiros das regiões N ordeste e N orte em comparação aos dados de referência.

No Brasil, os efeitos da Segunda Guerra Mundial na ocorrência deTSC foram investigados com base em dados de estatura de recrutas da M arinha nascidos entre 1940 e 1965. Observa-se que, no decorrer da primeira metade da década de quarenta, houveum declínio na média de estatura de $1,1 \mathrm{~cm}$. Esta queda nas médias de estatura foi atribuída aos efeitos da Segunda Guerra M undial. 0 argumento resideno fato dequea influência exercida pelo ambiente na determinação da estatura adulta ocorre principalmente nos primeiros anos de vida ${ }^{10,11}$. M esmo não estando envolvido diretamente na guerra, está claro que a conjuntura econômica foi desfavorável ao Brasil na época ${ }^{12}$. Isso pode ser uma das explicações para o fato do aumento de cinco centímetros na estatura final dos recrutas de Blumenau, quando se compara as estaturas finais dos nascidos no período da Segunda Guerra Mundial com os nascidos no período pós-guerra.

A observação da evolução da estatura em indivíduos aptos para o serviço militar (Exército) entre 1979 e 1991 em 24 estados brasileiros demonstra que ocorreu uma elevação na estatura média para a grande maioria dos estados. Os maiores incrementos para os indivíduos com 0 mais alto grau de escolaridade foram observados no Sergipe $(4,0 \mathrm{~cm})$, seguido de M inas Gerais (3,9 $\mathrm{cm})$ e M ato Grosso $(3,0 \mathrm{~cm})$. Não foram observados incrementos no Espírito Santo, Ceará, Amapá e $\mathrm{Acre}^{13}$. 0 maior incremento deste estudo pode ser comparado com o estudo de Blumenau econcluído quea população destemunicípio teve a mesma TSC de Sergipe, $4 \mathrm{~cm} /$ década.

Ao se analisar a TSC de 4.447 indivíduos alistados na Junta Militar de Rio Branco ( $A C$ ), entre
1948 e 1974, nota-se uma tendência positiva e o incremento médio em estatura foi de $0,07 \mathrm{~cm} /$ ano ${ }^{14}$. Já no atual estudo, comparando-se com a década de sessenta, em Blumenau, este município teve a tendência de $1 \mathrm{~cm} /$ década.

0 estudo realizado por Figueiró ${ }^{15}$ analisou a TSC em alistados no exército no estado de Pernambuco entre 1979 e 1990. A diferença na estatura entre o primeiro e o último ano estudado foi de $3,7 \mathrm{~cm}$ para o conjunto dos alistados. Se a comparação for feita com o estudo de Blumenau, verifica-se que, durante a década de oitenta, a população teve um ganho de quatro centíme tros na estatura final.

Nota-se que, assim como as literaturas sobre 0 assunto, neste período, na cidade de Blumenau, encontrou-setendência secular de crescimento em estatura positiva, tendo a população de Blumenau aumentado sete centímetros nos últimos cinquenta anos.

A análise dos dados não nos permitiu fazer qual quer comparação entre estatura final e grau de escolaridade ou estatura final e peso, como vários trabalhos publicados o fazem, visto que os dados de escolaridade e peso das fichas dos recrutas não eram informados na maioria delas. Entretanto, sem dúvida, ocorreram na cidade de Blumenau mudanças sociais, econômicas, em seu nível deatenção à saúde, educação eprestação de serviços, que pudessem justificar mudanças positivas na tendência secular de crescimento.

Desta forma, fica como sugestão para um outro trabalho a análise dos principais fatores responsáveispelatendência secular decrescimento em estatura ser positiva no município de Blumenau.

\section{Considerações finais}

A tendência secular do crescimento em estatura no município de Blumenau (SC) foi positiva nos últimos cinquenta anos.

0 val or das médias das alturas nas décadas de sessenta, setenta, oitenta, noventa e 2000 foi, respectivamente, $1,70 \mathrm{~m} ; 1,71 \mathrm{~m} ; 1,75 \mathrm{~m} ; 1,75 \mathrm{~m} ; 1,77 \mathrm{~m}$.

A taxa de ganho médio em estatura deste município foi desete centímetrosnosúltimoscinquenta anos, o que correspondea 1,4 cm/década ou $0,14 \mathrm{~cm} / \mathrm{ano}$. 


\section{Colaboradores}

AS Soncini, DM Vargas, M GL Arena eLFGL Arena participaram igualmente de todas as etapas da elaboração do artigo.

\section{Referências}

1. Marmo DB, Zambon M P, Morcillo AM, Guimarey LM. Tendência secular de crescimento em escolares de Paulínia, São Paulo-Brasil (1979/80 - 1993/ 94) Rev Assoc M ed Bras [periódico na Internet]. 2004 [acessado 2006 mai 12];50(4):[cerca de 5 p.]. Disponível em: http://www.scielo.br/pdf/ramb/ v50n4/22749.pdf

2. Monteiro CA, Conde WL. Tendência secular do crescimento pós-natal na cidade de São Paulo (19741996). Rev. Saude Publica [periódico na Internet]. 2000 [acessado $2007 \mathrm{abr}$ 16];34(6):[cerca de 11 p.]. Disponível em: http://www.scielo.br/pdf/rsp/ v34n6s0/3517.pdf

3. Malina RM. Research on secular trends in auxology. Anthropolgischer Anzeiger 1990; 48:209-227.

4. França Jr. I, Silva GR, Monteiro CA. Tendência secular da altura na idade adulta de crianças nascidas na cidade de São Paulo entre 1950 e 1976. Rev. Saude Publica [periódico na Internet] 2000 [acessado 2007 jan 16];34(6):[cerca de 6 p.]. Disponível em: http://www.scielo.br/pdf/rsp/v34n6s0/3523.pdf

5. Kac G. Tendência secular em estatura em recrutas da Marinha do Brasil nascidos entre 1940 e 1965. Cad Saude Publica [periódico na Internet] 1998 [acessado 2006 mar 16]; 14(3):[cerca de 9 p.]. Disponível em: http://www.scielo.br/pdf/csp/v14n3/ 0093.pdf

6. Kac G. Tendência secular em estatura: uma revisão da literatura. Cad Saude Publica [periódico na Internet] 1999 [acessado 2006 mar 06];15(3):[cerca de 11 p.]. Disponível em: http://www.scielo.br/pdf/csp/ v15n3/0484.pdf

7. Monteiro CA, Benicio MH, Gouveia NC. Secular Trends in Brazil Over Three Decades. Ann. Human. Biol. 1994; 21:381-390.

8. Meredith HV. Findings from Asia, Australia, Europe and N orth America on Secular Change in M ean $\mathrm{H}$ eight of Children and Young Adult. Am J. Phys Anthropol. 1976; 44:315-326.
9. Floud R. The heights of Europeans since 1750: A new source for European economic history. Cambridge, Massachusetts: National Bureau of Economic Research; 1984.

10. Waterlow JC. Observations on the natural history of stunting. In: Waterlow JC, editor. Linear growth retardation in less developed countries. Vevey: Nestle Nutrition; New York: Raven Press; 1988.

11. Tanner JM. Fetus into $M$ an. From Conception to Maturity. Cambridge: Harvard University Press; 1989.

12. Oliveira A, Lobo EML. O Estado Novo e o sindicato corporativista, 1937-1945. In: Lobo EM L, organizador. Rio de Janeiro operário: natureza do Estado, a conjuntura econômica, condições de vida e consciência de classe, 1930-1970. Rio de Janeiro: Access; 1992. p. 102-127.

13. Marcondes $E, M$ arques RM. Estudo antropométrico de indivíduos aptos para o serviço militar no período de 1979 a 1991. Rev. bras. crescimento desenvolv. hum. 1993; 2:9-38.

14. Paiva FCF. Estudo da tendência de crescimento dos jovens nascidos entre 1948 e 1974, alistados no serviço militar em Rio Branco - Acre. Rio Branco: Universidade Federal do Acre; 1994.

15. Figueiró AC. Perfil do crescimento dos jovens alistados no Exército no Estado de Pernambuco entre 1979 e 1990. [dissertação] Recife (PE): Departamento de Nutrição, Universidade Federal de Pernambuco; 1994.

Artigo apresentado em 03/08/2008

Aprovado em 28/01/2009

Versão final apresentada em 01/03/2009 